\title{
Hand-assisted laparoscopy for wandering spleen
}

\author{
Michael Cripps $\cdot$ Jonathan Svahn
}

Received: 23 April 2009/Accepted: 19 March 2010/Published online: 29 May 2010

(C) The Author(s) 2010. This article is published with open access at Springerlink.com

\begin{abstract}
A wandering spleen is a rare condition with an unknown incidence. The lack of short gastric vessels and suspensory ligaments is thought to result from a fusion anomaly of the dorsal mesogastrium of the spleen. The major risk in performing a splenectomy for patients with a wandering spleen is overwhelming postsplenectomy infection (OPSI). The incidence of OPSI is $0.13 \%$ to $8.1 \%$, with a mortality rate of $30 \%$ to $60 \%$. Laparoscopic splenopexy provides the benefits of minimally invasive surgery while avoiding the complications of splenectomy. The reported case presents a patient with a wandering spleen. The patient, an 18-year-old woman, experienced a malarial infection at the age of 5 years and had a computed tomography (CT) scan documenting her spleen in the normal anatomic position. When she was 18 years old, a CT scan showed her spleen located in the right lower quadrant (RLQ). At laparoscopy, the presence of the spleen in the RLQ was confirmed. The spleen could not be easily manipulated with laparoscopic instruments, so a hand port was used to mobilize the spleen to the left upper quadrant (LUQ). No evidence of attenuated
\end{abstract}

Electronic supplementary material The online version of this article (doi:10.1007/s00464-010-1120-0) contains supplementary material, which is available to authorized users.

Presented at the SAGES 2009 Annual Meeting, April 22-24, 2009, Phoenix, AZ.

M. Cripps

Department of Surgery, University of California San Francisco,

East Bay, Oakland, CA, USA

e-mail: michael.cripps@sbcglobal.net

J. Svahn $(\bowtie)$

Department of Surgery, Kaiser Permanente East Bay,

Oakland, CA, USA

e-mail: Jonathan.Svahn@kp.org suspensory ligaments was seen. The spleen was secured in the left subdiaphragmatic location by the use of a Vicryl mesh bag. An omental sling was used to support the spleen further. A 1-year follow up CT confirmed that the spleen still was located in the correct anatomic position. This is a unique case in that the patient was known to have a normally located spleen at a young age and then found to have a wandering spleen later in life. This could have resulted from a congenital fusion anomaly or attenuation of the patient's suspensory ligaments caused by her previous malarial infection and splenomegaly.

Keywords Hand-assisted laparoscopy .

Wandering spleen

Disclosure Michael Cripps, M. D. and Jonathan Svahn, M. D. have no conflicts of interest or financial ties to disclose.

Open Access This article is distributed under the terms of the Creative Commons Attribution Noncommercial License which permits any noncommercial use, distribution, and reproduction in any medium, provided the original author(s) and source are credited.

\section{Reference}

1. Kleiner O, Newman N, Cohen Z (2006) Pediatric wandering spleen successfully treated by laparoscopic splenopexy. J Laparoendosc Adv Surg Tech A 16(3):328-330 